\title{
Optomechanical-like coupling between superconducting resonators
}

\author{
J. R. Johansson, ${ }^{1, *}$ G. Johansson, ${ }^{2}$ and Franco Nori ${ }^{3,4}$ \\ ${ }^{1}$ iTHES Research Group, RIKEN, Wako-shi, Saitama 351-0198, Japan \\ ${ }^{2}$ Microtechnology and Nanoscience, MC2, Chalmers University of Technology, SE-412 96 Göteborg, Sweden \\ ${ }^{3}$ CEMS, RIKEN, Wako-shi, Saitama 351-0198, Japan \\ ${ }^{4}$ Department of Physics, University of Michigan, Ann Arbor, Michigan 48109-1040, USA
}

(Received 4 April 2014; published 18 November 2014)

\begin{abstract}
We propose and analyze a circuit that implements a nonlinear coupling between two superconducting microwave resonators. The resonators are coupled through a superconducting quantum interference device that terminates one of the resonators. This produces a nonlinear interaction of the standard optomechanical form, where the quadrature of one resonator couples to the photon number of the other resonator. The circuit therefore allows for all-electrical realizations of analogs to optomechanical systems, with coupling that can be both strong and tunable. We estimate the coupling strengths that should be attainable with the proposed device, and we find that the device is a promising candidate for realizing the single-photon strong-coupling regime. As a potential application, we discuss implementations of networks of nonlinearly coupled microwave resonators, which could be used in microwave-photon-based quantum simulation.
\end{abstract}

DOI: 10.1103/PhysRevA.90.053833

PACS number(s): 42.50.Pq, 42.50.Wk, 85.25.Cp

\section{INTRODUCTION}

Superconducting microwave resonators have emerged as one of the key components in quantum electronics [1-4] in recent years. In a parallel development, the field of quantum optomechanics [5-7] have seen equally impressive progress, with recent accomplishments including sideband cooling of mechanical resonators to their ground state [8,9], normal-mode splitting [10-12], generation of nonclassical states of light $[13,14]$, near quantum-limited detection [15-17], and state transfer [18,19]. In several of these recent works [8,11,15-19], microwave resonators, rather than optical cavities, were coupled to the mechanical systems. Meanwhile, in electrical systems, superconducting microwave resonators have been used as quantum buses to couple superconducting qubits in a variety of architectures [20-22], for readout and control of superconducting qubits [23-26], for characterization of quantum dots [27-30], and for interfacing different types of quantum systems in hybrid circuits [31].

Coupled microwave resonators has also been studied extensively, both theoretically [32-34] and experimentally [35]. However, in these circuits, the resonators are typically coupled linearly to each other or to other quantum systems, through the amplitude of the resonator's electric or magnetic field. Here we investigate a nonlinear coupling between two microwave resonators [36], where, in a certain regime, the field amplitude in one resonator couples to the photon number in the other resonator. This is exactly the type of interaction encountered in optomechanical systems [5-7], making it possible to implement analogs of optomechanical systems in all-electrical circuits. In such analogs, the mechanical component is replaced by an electrical resonator, but without losing the interesting nonlinear coupling that is characteristic for optomechanical systems. Moreover, using this type of device, it appears possible to reach the single-photon strong-coupling regime. This regime has recently received

\footnotetext{
*robert@ riken.jp
}

considerable attention, and a number of interesting phenomena has been theoretically predicted, including photon blockade effects [37], multiple cooling resonances [38,39], and the generation of nonclassical states [40-43].

The physical realization of this nonlinear coupling uses a superconducting quantum interference device (SQUID) embedded in one of the resonators. The magnetic flux that threads the SQUID loop can modify the properties of the resonator, such as its resonance frequency [44]. Superconducting microwave resonators with embedded SQUIDs have been proposed for realizing tunable [36] and beam-splitter [45] coupling, and have been used to implement frequency-tunable resonators [46-48] with tunable boundary conditions and tunable index of refraction. With parametrically modulated applied magnetic flux, i.e., with classical driving fields, these types of devices have been used to implement parametric amplifiers [48-50] and nonadiabatic quantum phenomena such as the dynamical Casimir effect [51-55]. See, e.g., Ref. [56] for a recent review.

Here we are interested in the case when the applied magnetic flux through the SQUID is due to the quantum field of another superconducting resonator, i.e., a quantized drive field. Also, we consider the situation where the modulated resonator adiabatically adjusts to the changes imposed by the magnetic flux through the embedded SQUID. Under these conditions we can formulate an effective Hamiltonian that describes the dynamics of the system. We show that this effective Hamiltonian is of the standard optomechanical form.

The remaining part of this paper is organized as follows. In Sec. II we introduce the device and set up a model for it. Here we use the Lagrangian formalism to model a lump-element representation of the circuit to obtain the boundary conditions and find the adiabatic mode functions for the resonators. In Sec. III we use the derived mode functions to formulate an effective Hamiltonian for the system, which is shown to be of the optomechanical form in Sec. III A. In Sec. IV we analyze possible coupling designs and evaluate the corresponding coupling strengths, and in Sec. V we discuss dissipation and the decay rates of the two resonators. In Sec. VI we discuss 
possible circuit layouts for realizing arrays of nonlinearly coupled resonators. Finally, we summarize our results in Sec. VII.

\section{DEVICE AND ITS CIRCUIT MODEL}

The type of device we investigate here is shown in one possible configuration in Fig. 1. Alternative configurations could also be used, with for example a SQUID located in the middle of resonator $A$, or with resonator $A$ made of an array of SQUIDs. The main properties of the system would remain unchanged.

Here we focus on a quantum mechanical analysis of the device in Fig. 1. The flux through the SQUID can in a certain regime be seen as modulating the effective length of resonator $A$ or, equivalently, its fundamental resonance frequency $\omega_{A}$. The flux through the SQUID is partly due to the magnetic field generated by resonator $B$. We therefore expect an interaction of the form $a^{\dagger} a\left(b+b^{\dagger}\right)$, where $a$ and $b$ are the annihilation operators for resonator $A$ and $B$, respectively. In the following we derive this result from a detailed quantum network analysis $[57,58]$ of the circuit.

\section{A. Circuit Lagrangian}

We model the electrical circuit in Fig. 1 by decomposing it in lumped-circuit elements, as shown, for the most relevant part of the circuit, in Fig. 2. As generalized coordinates we use the magnetic node fluxes $\Phi_{n}^{\alpha}$ (where $\alpha=A, B$ ), which are related to the node voltages $V_{n}^{\alpha}$ as $\Phi_{n}^{\alpha}=\int^{t} d t^{\prime} V_{n}^{\alpha}\left(t^{\prime}\right)$, and to the gauge-invariant superconducting node phases $\varphi_{n}^{\alpha}=$ $2 \pi \Phi_{n}^{\alpha} / \Phi_{0}$. In terms of these coordinates, the Lagrangian of

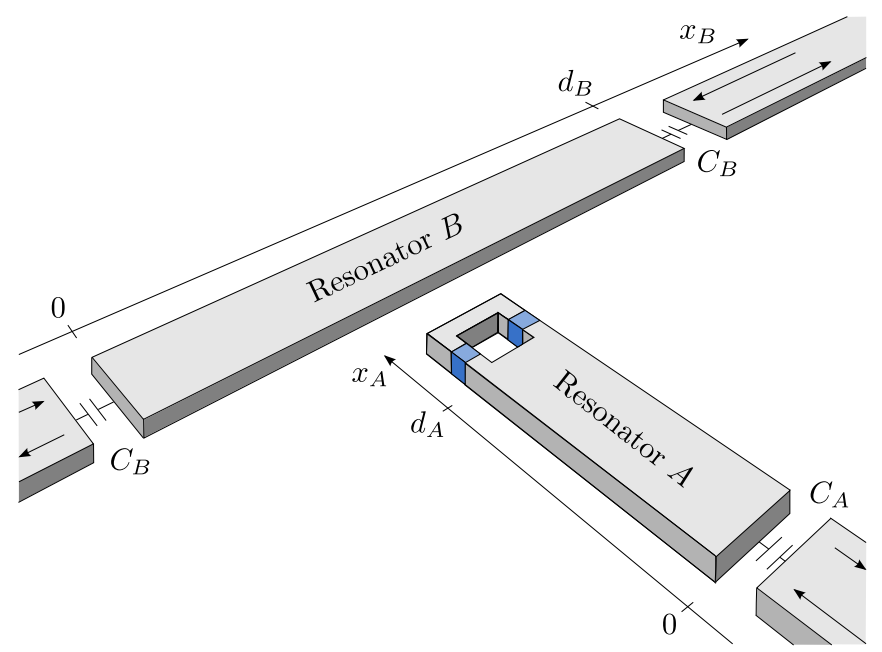

FIG. 1. (Color online) Schematic illustration of the system, which consists of two superconducting transmission-line resonators $A$ and $B$. The resonators are coupled to each other through the SQUID terminating resonator $A$. The coupling mechanism is the following: part of the magnetic field generated by the signal in resonator $B$ threads the SQUID loop that terminates resonator $A$, changing the phase across the SQUID. This phase determines the boundary condition for resonator $A$. The result is an interaction where the amplitude of resonator $B$ couples to the photon number of resonator $A$.

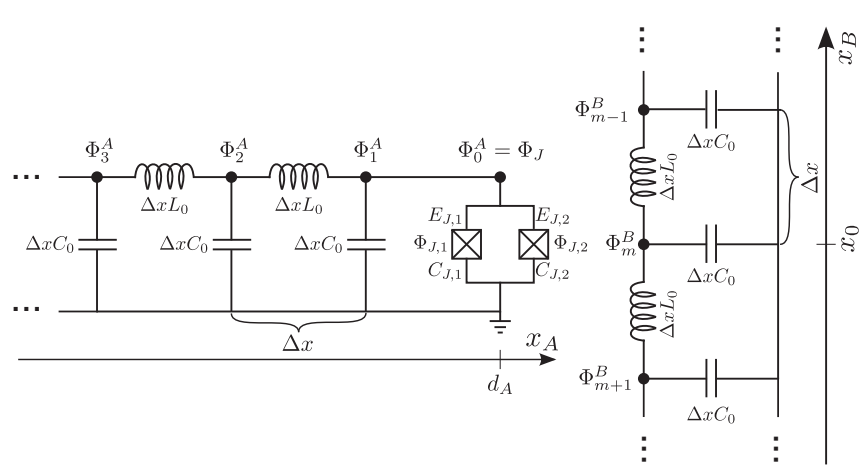

FIG. 2. Circuit diagram of the coupler part of the circuit in Fig. 1, where resonator $B$ meets the SQUID embedded in resonator $A$.

the circuit can be written as

$$
\mathcal{L}=\mathcal{L}_{A}+\mathcal{L}_{B}+\mathcal{L}_{S}
$$

where

$$
\begin{gathered}
\mathcal{L}_{\alpha}=\frac{1}{2} \sum_{n=1}^{N_{\alpha}}\left(\Delta x C_{0}^{\alpha}\left(\dot{\Phi}_{n}^{\alpha}\right)^{2}-\frac{\left(\Phi_{n+1}^{\alpha}-\Phi_{n}^{\alpha}\right)^{2}}{\Delta x L_{0}^{\alpha}}\right) \\
\mathcal{L}_{\mathrm{S}}=\frac{1}{2} C_{J}\left(\dot{\Phi}_{J}\right)^{2}+E_{J}\left(\Phi_{\mathrm{ext}}\right) \cos \left(2 \pi \frac{\Phi_{J}}{\Phi_{0}}\right) .
\end{gathered}
$$

Here we have assumed that the SQUID is symmetric $\left(C_{J, i}=\right.$ $C_{J} / 2$ and $\left.E_{J, i}=E_{J}\right)$ and we have written its Lagrangian $\mathcal{L}_{\mathrm{S}}$ of the form of an effective Josephson junction with Josephson energy

$$
E_{J}\left(\Phi_{\text {ext }}\right)=2 E_{J}\left|\cos \left(\pi \frac{\Phi_{\text {ext }}}{\Phi_{0}}\right)\right| .
$$

In the following we also assume that the two transmission lines are identical, with $C_{0}^{\alpha}=C_{0}$ and $L_{0}^{\alpha}=L_{0}$.

From the circuit Lagrangian, we obtain equations of motion for flux nodes $\Phi_{n}^{\alpha}$. In the continuum limit, $\Delta x \rightarrow 0$, the resulting flux fields $\Phi_{A}(x, t)$ and $\Phi_{B}(x, t)$ are found to obey the one-dimensional massless Klein-Gordon wave equation, which has a continuum of independent plane-wave solutions propagating in the positive and negative direction, respectively. We can therefore write the quantum-mechanical representation of the flux field as

$$
\begin{aligned}
\Phi(x)= & \sqrt{\frac{Z_{0} \hbar}{4 \pi}} \int_{-\infty}^{\infty} \frac{d \omega}{\sqrt{|\omega|}}\left[a_{R}(\omega) e^{-i\left(-k_{\omega} x+\omega t\right)}\right. \\
& \left.+a_{L}(\omega) e^{-i\left(k_{\omega} x+\omega t\right)}\right]
\end{aligned}
$$

where $a_{L}(\omega)$ and $a_{R}(\omega)$ are annihilation operators for the fields propagating in the negative and positive $x$ direction, respectively, satisfying the commutation relation $\left[a_{L}(\omega), a_{L}^{\dagger}\left(\omega^{\prime}\right)\right]=\left[a_{R}(\omega), a_{R}^{\dagger}\left(\omega^{\prime}\right)\right]=\delta\left(\omega-\omega^{\prime}\right)$, and $a_{L}(-\omega)=a_{L}^{\dagger}(\omega), a_{R}(-\omega)=a_{R}^{\dagger}(\omega)$. Here, $Z_{0}=\sqrt{L_{0} / C_{0}}$ is the characteristic impedance, $k_{\omega}=\omega / v$ is the wave number, and $v=1 / \sqrt{L_{0} C_{0}}$ the propagation speed of the signal in the transmission line. At the boundaries, i.e., at $x=0$ and $x=d_{A}$ for resonator $A$, and at $x=0$ and $x=d_{B}$ for resonator $B$ (see Fig. 1), the equations of motion define the boundary conditions for the continuum fields $\Phi_{A}(x)$ and $\Phi_{B}(x)$. These boundary 
conditions can be used to derive the mode functions for the resonators.

\section{B. Boundary conditions}

In this section we write down the boundary conditions for the two sides of the two resonators. Here we assume that the two resonators have well-defined resonance frequencies, i.e., their quality factors are high, and the capacitive coupling to the external transmission lines shown in Fig. 1 can be neglected (that is, we consider the limit $C_{A, B} \rightarrow 0$ ). In this limit, the boundary conditions therefore correspond to that of an open-ended resonator. Finite capacitances $C_{A, B}$ result in a broadening and small frequency shifts of each mode (see, e.g., Ref. [52], and also Sec. V, where we further discuss dissipation and the decay rates of the resonators).

\section{Resonator $A$}

In the limit $C_{A} \rightarrow 0$, resonator $A$ is open ended at $x=0$ (see Fig. 1), and the corresponding boundary condition is

$$
\partial_{x} \Phi_{A}(0, t)=0 .
$$

At the end terminated by the SQUID, $x=d_{A}$, the boundary condition $[44,52]$ can be written as

$$
\begin{gathered}
C_{J} \partial_{t t} \Phi_{A}\left(d_{A}, t\right)+\left(\frac{2 \pi}{\Phi_{0}}\right)^{2} E_{J}\left(\Phi_{\mathrm{ext}}\right) \Phi_{A}\left(d_{A}, t\right) \\
+\frac{1}{L_{0}} \partial_{x} \Phi_{A}\left(d_{A}, t\right)=0 .
\end{gathered}
$$

In general, the SQUID that terminates resonator $A$ is a dynamical system of its own, and its energy-level spectrum is anharmonic due to the $\cos \left(\Phi_{J}\right)$ dependence in its potential. However, in the considered parameter regime where $\Phi_{J} / \Phi_{0}<1$, the nonlinearity is small and the first excited state is characterized by the SQUIDs plasma frequency $\omega_{p}=$ $2 \pi \sqrt{E_{J} / \Phi_{0}^{2} C_{J}}$. If the plasma frequency of the SQUID is large compared to the frequencies of the relevant modes in resonator $A$, then the SQUID remains adiabatically in its ground state and can be described as a lumped-circuit element. In experiments with SQUID-terminated transmission lines [47,54], the plasma frequency of the SQUID can be made relatively high $(\sim 70 \mathrm{GHz}$ or higher), which is large compared to the typical frequencies of photons in the transmission line (order of $10 \mathrm{GHz}$, or less). These studies have experimentally demonstrated that the lumped-circuit element model of the SQUID in this type of system can be a good approximation. In this case, we can neglect the first term in the boundary condition Eq. (7), and write

$$
\Phi_{A}\left(d_{A}, t\right)+\Delta d\left(\Phi_{\text {ext }}\right) \partial_{x} \Phi_{A}\left(d_{A}, t\right)=0
$$

where

$$
\Delta d\left(\Phi_{\text {ext }}\right)=\left(\frac{\Phi_{0}}{2 \pi}\right)^{2} \frac{1}{L_{0} E_{J}\left(\Phi_{\text {ext }}\right)} .
$$

This can be interpreted as an effective length that can be tuned by the externally applied magnetic flux $\Phi_{\text {ext }}$. If this effective length is small compared to the length scale at which $\Phi_{A}(x)$ varies substantially, i.e., small compared to the wavelength, then Eq. (8) can be viewed as a differential. If we imagine that the transmission line uniformly extends beyond the point $x=d_{A}$ for an additional length $\Delta d\left(\Phi_{\text {ext }}\right)$, we can then rewrite the boundary condition Eq. (8) on the simple form

$$
\Phi_{A}\left(d_{\text {eff }}\left(\Phi_{\text {ext }}\right), t\right)=0,
$$

where we have introduced the new effective tunable length of resonator $A$

$$
d_{\text {eff }}\left(\Phi_{\text {ext }}\right)=d_{A}+\Delta d\left(\Phi_{\text {ext }}\right)
$$

\section{Resonator B}

In the limit $C_{B} \rightarrow 0$, resonator $B$ is open ended at both $x=$ 0 and $x=d_{B}$ (see Fig. 1), and the corresponding boundary conditions are therefore

$$
\begin{gathered}
\partial_{x} \Phi_{B}(0, t)=0, \\
\partial_{x} \Phi_{B}\left(d_{B}, t\right)=0 .
\end{gathered}
$$

\section{SQUID biasing and effective length}

The externally applied magnetic flux, $\Phi_{\text {ext }}$, is partly produced by the field of resonator $B$, and partly by a static background flux, $\Phi_{\mathrm{ext}}^{0}$. Here we assume that the physical dimension of SQUID loop is small compared to the typical length scale at which the field in resonator $B$ varies. We can then decompose the externally applied magnetic flux in a static bias and a small deviation,

$$
\Phi_{\mathrm{ext}}=\Phi_{\mathrm{ext}}^{0}+\Delta \Phi_{\mathrm{ext}}
$$

where the small deviation $\Delta \Phi_{\text {ext }}$ is a function of the field amplitude at a single point $x_{0}$ in resonator $B$. For now we are not concerned with the detailed form of $\Delta \Phi_{\text {ext }}$, and we only require it to be small compared to $\Phi_{0}$. Under this condition we can expand the effective Josephson energy of the SQUID, Eq. (4), for $\Phi_{\text {ext }}^{0} \in\left[0, \Phi_{0} / 2\right]$, as

$$
E_{J}\left(\Phi_{\mathrm{ext}}\right) \approx E_{J}^{0}-2 E_{J} \frac{\pi}{\Phi_{0}} \Delta \Phi_{\mathrm{ext}} \sin \left(\pi \frac{\Phi_{\mathrm{ext}}^{0}}{\Phi_{0}}\right),
$$

where

$$
E_{J}^{0}=2 E_{J}\left|\cos \left(\pi \frac{\Phi_{\mathrm{ext}}^{0}}{\Phi_{0}}\right)\right| .
$$

Using Eq. (15) in the expression for the effective length associated with the SQUID, Eq. (9), we obtain

$$
\Delta d\left(\Phi_{\mathrm{ext}}\right) \approx\left(\frac{\Phi_{0}}{2 \pi}\right)^{2} \frac{1}{L_{0} E_{J}^{0}}\left[1+\pi \frac{\Delta \Phi_{\mathrm{ext}}}{\Phi_{0}} \tan \left(\pi \frac{\Phi_{\mathrm{ext}}^{0}}{\Phi_{0}}\right)\right],
$$

and to simplify the expressions we write

$$
\Delta d\left(\Phi_{\text {ext }}\right)=\Delta d_{0}\left(\Phi_{\text {ext }}^{0}\right)+\delta d\left(\Phi_{\text {ext }}^{0}\right) \Delta \Phi_{\text {ext }},
$$

with

$$
\begin{gathered}
\Delta d_{0}\left(\Phi_{\mathrm{ext}}^{0}\right)=\left(\frac{\Phi_{0}}{2 \pi}\right)^{2} \frac{1}{L_{0} E_{J}^{0}}, \\
\delta d\left(\Phi_{\mathrm{ext}}^{0}\right)=\frac{1}{2}\left(\frac{\Phi_{0}}{2 \pi}\right) \frac{1}{L_{0} E_{J}^{0}} \tan \left(\pi \frac{\Phi_{\mathrm{ext}}^{0}}{\Phi_{0}}\right),
\end{gathered}
$$


and

$$
\begin{gathered}
d_{\mathrm{eff}}\left(\Phi_{\mathrm{ext}}\right)=d_{\mathrm{eff}}^{0}\left(\Phi_{\mathrm{ext}}^{0}\right)+\delta d\left(\Phi_{\mathrm{ext}}^{0}\right) \Delta \Phi_{\mathrm{ext}}, \\
d_{\mathrm{eff}}^{0}\left(\Phi_{\mathrm{ext}}^{0}\right)=d_{A}+\Delta d_{0}\left(\Phi_{\mathrm{ext}}^{0}\right) .
\end{gathered}
$$

\section{Fields and modes}

Given the quantum description of the flux field in the two resonators given in Eq. (5), we are now interested in using the boundary conditions given in the previous section to derive the adiabatic modes for the two resonators.

\section{Resonator A}

With the two boundary conditions, Eqs. (6) and (7), corresponding to an open and a short circuit, respectively, resonator $A$ becomes a $\lambda / 4$ resonator. In particular, imposing the two boundary conditions results in the constraint $\cos \left[k_{\omega}^{A} d_{\text {eff }}\left(\Phi_{\text {ext }}\right)\right]=0$, which is satisfied with the frequencies $\omega_{n}^{A}=\frac{\pi}{2}(2 n+1) v / d_{\text {eff }}\left(\Phi_{\text {ext }}\right)$. The field, written in terms of the corresponding discrete mode functions, becomes

$$
\begin{aligned}
\Phi_{A}(x, t)= & \sqrt{\frac{Z_{0} \hbar}{2 \pi}} \sum_{n} \sqrt{\frac{\omega_{d_{A}}}{\omega_{n}^{A}}} \cos \left(\frac{\pi(2 n+1) x}{2 d_{\text {eff }}\left(\Phi_{\text {ext }}\right)}\right) \\
& \times\left(a_{n} e^{-i \omega_{n}^{A} t}+\text { H.c. }\right),
\end{aligned}
$$

where $\omega_{d_{A}}=2 \pi v / d_{\text {eff }}\left(\Phi_{\text {ext }}\right)$ is the full-wavelength frequency of the resonator of length $d_{\mathrm{eff}}\left(\Phi_{\mathrm{ext}}\right)$, and $a_{n}$ is the annihilation operator of the $n$th mode, which satisfies $\left[a_{n}, a_{m}^{\dagger}\right]=\delta_{n m}$. Here the field is written in terms of the instantaneous, or adiabatic, mode functions for resonator $A$, for a given applied magnetic flux $\Phi_{\text {ext }}$.

\section{Resonator $B$}

With the two boundary conditions, Eqs. (12) and (13), which both are open-ended terminations, resonator $B$ becomes a $\lambda / 2$ resonator. In particular, imposing these two boundary conditions results in the constraint $\sin \left(k_{\omega}^{B} d_{B}\right)=0$, which is satisfied with the frequencies $\omega_{n}^{B}=\pi n v / d_{B}$. Writing the field in terms of the corresponding discrete mode functions yields

$$
\begin{aligned}
\Phi_{B}(x, t)= & \sqrt{\frac{Z_{0} \hbar}{2 \pi}} \sum_{n} \sqrt{\frac{\omega_{d_{B}}}{\omega_{n}^{B}}} \cos \left(\frac{\pi n x}{d_{B}}\right) \\
& \times\left(b_{n} e^{-i \omega_{n}^{B} t}+\text { H.c. }\right),
\end{aligned}
$$

where $\omega_{d_{B}}=2 \pi v / d_{B}$, and $b_{n}$ is the annihilation operator of the $n$th mode, satisfying $\left[b_{n}, b_{m}^{\dagger}\right]=\delta_{n m}$.

\section{EFFECTIVE HAMILTONIAN}

Using the adiabatic modes derived in the previous section, and their corresponding annihilation operators, we can write the Hamiltonian for the two resonators of the form

$$
H=\sum_{n} \hbar \omega_{n}^{A} a_{n}^{\dagger} a_{n}+\sum_{n} \hbar \omega_{n}^{B} b_{n}^{\dagger} b_{n} .
$$

Assuming that $\delta d\left(\Phi_{\text {ext }}^{0}\right) \Delta \Phi_{\text {ext }} \ll d_{\text {eff }}^{0}\left(\Phi_{\text {ext }}\right)$, which implies that the optical length modulation is small compared to the total optical length of resonator $A$, we can now use Eq. (21) to write the mode frequencies for resonator $A$ as

$$
\begin{aligned}
\omega_{n}^{A} & =\frac{\pi}{2} \frac{(2 n+1) v}{d_{\mathrm{eff}}^{0}\left(\Phi_{\mathrm{ext}}^{0}\right)+\delta d\left(\Phi_{\mathrm{ext}}^{0}\right) \Delta \Phi_{\mathrm{ext}}} \\
& \approx \tilde{\omega}_{n}^{A}\left(1-\frac{\delta d\left(\Phi_{\mathrm{ext}}^{0}\right)}{d_{\mathrm{eff}}^{0}\left(\Phi_{\mathrm{ext}}^{0}\right)} \Delta \Phi_{\mathrm{ext}}\right),
\end{aligned}
$$

where $\tilde{\omega}_{n}^{A}=\frac{\pi}{2}(2 n+1) v / d_{\text {eff }}^{0}\left(\Phi_{\text {ext }}^{0}\right)$. Inserting this expression in the Hamiltonian Eq. (25), we obtain an effective Hamiltonian

$$
\begin{aligned}
H= & \sum_{n} \hbar \tilde{\omega}_{n}^{A} a_{n}^{\dagger} a_{n}+\sum_{n} \hbar \omega_{n}^{B} b_{n}^{\dagger} b_{n} \\
& -\sum_{n} \hbar \omega_{n}^{A} \frac{\delta d\left(\Phi_{\mathrm{ext}}^{0}\right)}{d_{\mathrm{eff}}^{0}\left(\Phi_{\mathrm{ext}}^{0}\right)} \Delta \Phi_{\mathrm{ext}} a_{n}^{\dagger} a_{n} .
\end{aligned}
$$

This Hamiltonian is valid when the modes of resonator $A$ instantaneously adjust to changes in the applied magnetic flux $\Delta \Phi_{\text {ext }}$, which are due to the dynamics of the field in resonator $B$. This is the case for the lowest few modes of resonator $A$ when $\omega_{A} \gg \omega_{B}$, and when resonator $B$ is not very strongly excited. In this case, the optical length modulation is small compared to the wavelength of the relevant modes in $A$, and the round-trip time of the electromagnetic signal in resonator $A$ is much shorter than the time scale of variation in resonator $B$. The retardation effects due the changing boundary condition of resonator $A$ can then be neglected [59]. This adiabatic approximation thus requires that the frequency shift due to the third term in Eq. (27) is small compared to the resonance frequencies $\omega_{n}^{A}$.

We now assume that the deviation of the external bias flux from the static bias $\Phi_{\text {ext }}^{0}$ takes the form

$$
\Delta \Phi_{\mathrm{ext}}=\Phi_{0} \sum_{n} G_{n}\left(b_{n}+b_{n}^{\dagger}\right),
$$

where $G_{n}$ is the effective coupling strength between the $n$th mode and the SQUID, including for example geometric factors, and the normalized mode amplitude at the point of the SQUID. This form will be motivated later when explicit coupling geometries are considered. With this form of $\Delta \Phi_{\text {ext }}$, the effective Hamiltonian takes the form

$$
\begin{aligned}
H= & \sum_{n} \hbar \tilde{\omega}_{n}^{A} a_{n}^{\dagger} a_{n}+\sum_{n} \hbar \omega_{n}^{B} b_{n}^{\dagger} b_{n} \\
& -\sum_{n} \hbar \tilde{\omega}_{n}^{A} \frac{\delta d\left(\Phi_{\mathrm{ext}}^{0}\right)}{d_{\mathrm{eff}}^{0}\left(\Phi_{\mathrm{ext}}^{0}\right)} \Phi_{0} \sum_{m} G_{m}\left(b_{m}+b_{m}^{\dagger}\right) a_{n}^{\dagger} a_{n} .
\end{aligned}
$$

\section{A. Optomechanical Hamiltonian}

If we restrict the dynamics of the system to only involve the two fundamental modes (i.e., by not exciting any higher modes), we obtain a simplified two-mode Hamiltonian

$$
H=\hbar \omega_{A} a^{\dagger} a+\hbar \omega_{B} b^{\dagger} b-\hbar g_{0} a^{\dagger} a\left(b+b^{\dagger}\right),
$$

where, for brevity, we have dropped the indices on the annihilation operators and the mode frequencies. Here

$$
g_{0}=\omega_{A} F\left(\Phi_{\text {ext }}^{0}\right) G_{1}
$$


is the coupling strength between the two resonators, and

$$
F\left(\Phi_{\mathrm{ext}}^{0}\right)=\Phi_{0} \frac{\delta d\left(\Phi_{\mathrm{ext}}^{0}\right)}{d_{\mathrm{eff}}^{0}\left(\Phi_{\mathrm{ext}}^{0}\right)} .
$$

The coupling strength is comprised of two factors, in addition to the frequency factor $\omega_{A}$ : (i) a factor $F\left(\Phi_{\text {ext }}^{0}\right)$ that depends on the properties and the bias conditions of the SQUID, and (ii) a factor $G_{1}$ that depends on the geometric arrangement of the SQUID and the resonators. To produce a large coupling strength we are interested in maximizing both of these factors.

The Hamiltonian Eq. (30) is of the standard optomechanical form, and the device we consider here is therefore analogous with an optomechanical system. However, in contrast to an optomechanical system, here both resonators are electrical and the fundamental nonlinear interaction strength $g_{0}$ can be tuned by changing the flux bias $\Phi_{\text {ext }}^{0}$.

As in the optomechanical case [6], we have in the derivation of Hamiltonian Eq. (30) assumed that $\omega_{A} \gg \omega_{B}$, so that the field in resonator $A$ adiabatically adjusts to the parametrically changing resonance frequency due to the dynamics of resonator $B$. We can compensate for the difference in frequencies by applying a driving field on resonator $A$, with frequency $\omega_{d}$ and amplitude $\epsilon_{A}$,

$$
\begin{aligned}
H= & \hbar \omega_{A} a^{\dagger} a+\hbar \omega_{B} b^{\dagger} b-\hbar g_{0} a^{\dagger} a\left(b^{\dagger}+b\right) \\
& +\hbar\left(\epsilon_{A} a e^{-i \omega_{d} t}+\epsilon_{A}^{*} a^{\dagger} e^{i \omega_{d} t}\right),
\end{aligned}
$$

and applying the unitary transformation $U=\exp \left[i \omega_{d} a^{\dagger} a t\right]$, which makes the drive terms time independent,

$$
\begin{aligned}
H= & \hbar \Delta_{A} a^{\dagger} a+\hbar \omega_{B} b^{\dagger} b-\hbar g_{0} a^{\dagger} a\left(b^{\dagger}+b\right) \\
& +\hbar\left(\epsilon_{A} a+\epsilon_{A}^{*} a^{\dagger}\right) .
\end{aligned}
$$

Here $\Delta_{A}=\omega_{A}-\omega_{d}$, and if we choose $\Delta_{A}=\omega_{B}$, i.e., $\omega_{d}=\omega_{A}-\omega_{B}$, the two resonators are effectively resonant. Furthermore, if the amplitude of the applied driving field $\epsilon_{A}$ is large, we can linearize the coupling by applying the unitary displacement transformation $D(\alpha)=\exp \left[\alpha a^{\dagger}-\alpha^{*} a\right]$, where $\alpha=\epsilon_{A} / \Delta_{A}$, and neglecting the term $\hbar g_{0} a^{\dagger} a\left(b^{\dagger}+b\right)$. The linearized Hamiltonian is

$$
\begin{aligned}
H= & \hbar \Delta_{A} a^{\dagger} a+\hbar \omega_{B} b^{\dagger} b+\hbar g_{0} \alpha\left(a+a^{\dagger}\right)\left(b+b^{\dagger}\right) \\
& -\hbar g_{0}|\alpha|^{2}\left(b+b^{\dagger}\right),
\end{aligned}
$$

and here the strength of the effective linear coupling, $g_{0} \alpha$, is proportional to the driving amplitude. This is commonly used in optomechanics to enhance the coupling strength when the fundamental coupling strength $g_{0}$ itself is too small. This linear coupling regime has several important applications [6,7], including state transfer, sideband cooling, and parametric amplification. Also, in hybrid electro-optomechanical systems, it has been shown that strong Kerr nonlinearities can be realized in this weak-coupling regime [60].

If, on the other hand, the fundamental coupling strength $g_{0}$ is comparable to $\omega_{B}$, it is instructive to apply the unitary transformation $U=\exp \left[-g_{0} a^{\dagger} a\left(b^{\dagger}-b\right) / \omega_{B}\right]$, after which the Hamiltonian Eq. (30) takes the form

$$
H=\hbar \Delta_{A} a^{\dagger} a+\hbar \omega_{B} b^{\dagger} b+\hbar \frac{g_{0}^{2}}{\omega_{B}}\left(a^{\dagger} a\right)^{2} .
$$

This Hamiltonian includes a nonlinear Kerr term, i.e., an effective photon-photon interaction term, with coupling strength $g_{0}^{2} / \omega_{B}$. This regime has recently been actively studied theoretically in optomechanics [37-39], and it has been shown to feature interesting phenomena, such as photon blockade effects [37], and multiple cooling resonances [39], and allowing for the generation of nonclassical states [40-43].

\section{COUPLING STRENGTH}

In this section, we explicitly evaluate the coupling strength $g_{0}$ for two possible coupling geometries. We first turn our attention to the factor $F\left(\Phi_{\text {ext }}\right)$, which only depends on the properties and the bias conditions of the SQUID. The explicit form of $F\left(\Phi_{\text {ext }}\right)$ is

$$
F\left(\Phi_{\text {ext }}\right)=\pi \frac{\Delta d_{0}\left(\Phi_{\text {ext }}\right)}{d_{A}+\Delta d_{0}\left(\Phi_{\text {ext }}\right)} \tan \left(\pi \frac{\Phi_{\text {ext }}}{\Phi_{0}}\right),
$$

which is shown graphically in Fig. 3. It is clear that $F\left(\Phi_{\text {ext }}\right)$ can be made small by tuning $\Phi_{\text {ext }}$ to zero, and also that it can be tuned to the order of unity, or even much larger, by letting $\Phi_{\text {ext }}$ approach $\frac{1}{2} \Phi_{0}$. However, when $\Phi_{\text {ext }}$ approach $\frac{1}{2} \Phi_{0}$, the plasma frequency of the SQUID decrease rapidly, and an assumption in deriving the effective Hamiltonian was that this plasma frequency must be much larger than the resonance frequency $\omega_{A}$. This prohibits tuning $\Phi_{\text {ext }}$ too close to $\frac{1}{2} \Phi_{0}$. However, with reasonable values of $\Phi_{\text {ext }} / \Phi_{0}$ it is realistic to obtain $F\left(\Phi_{\text {ext }}\right)$ of the order of 1 (e.g., $\left.\Phi_{\text {ext }} / \Phi_{0} \approx 0.3-0.4\right)$. With an increased plasma frequency of the SQUID, i.e., a large Josephson energy $E_{J}, \Phi_{\text {ext }}$ could possibly be further increased.

In addition to the factor $F\left(\Phi_{\text {ext }}\right)$, the coupling strength Eq. (31) also contains the factor $G_{1}$, which depends on the detailed geometry of the coupling. Below we estimate the numerical values of $G_{1}$ for two possible geometries shown in Fig. 4.

\section{A. Inductive coupling}

A schematic illustration of a coupling design where the magnetic field of resonator $B$ couples inductively to the SQUID loop is shown in Fig. 4(a). An exact calculation of the coupling strength for this design would require detailed modeling of how the magnetic field extends around the

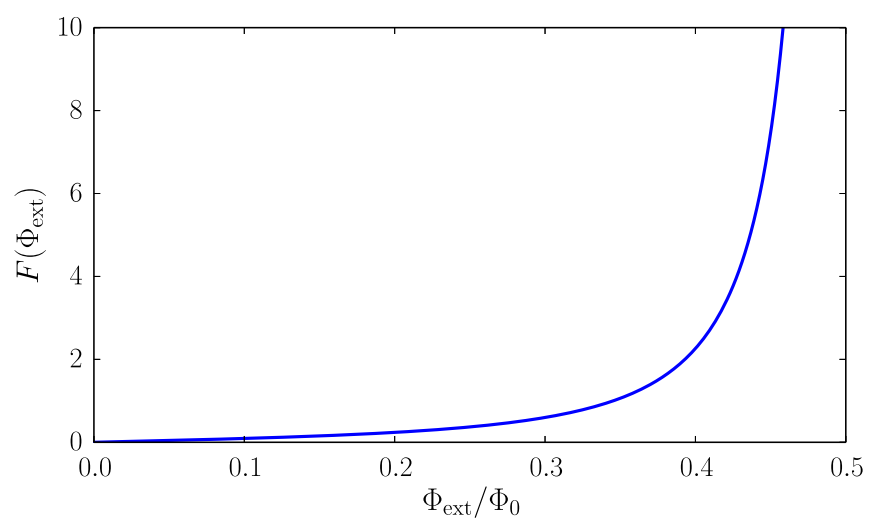

FIG. 3. (Color online) $\Phi_{\text {ext }}$ dependence of the factor $F\left(\Phi_{\text {ext }}\right)$ that appears in the coupling strength [see Eq. (31)]. 


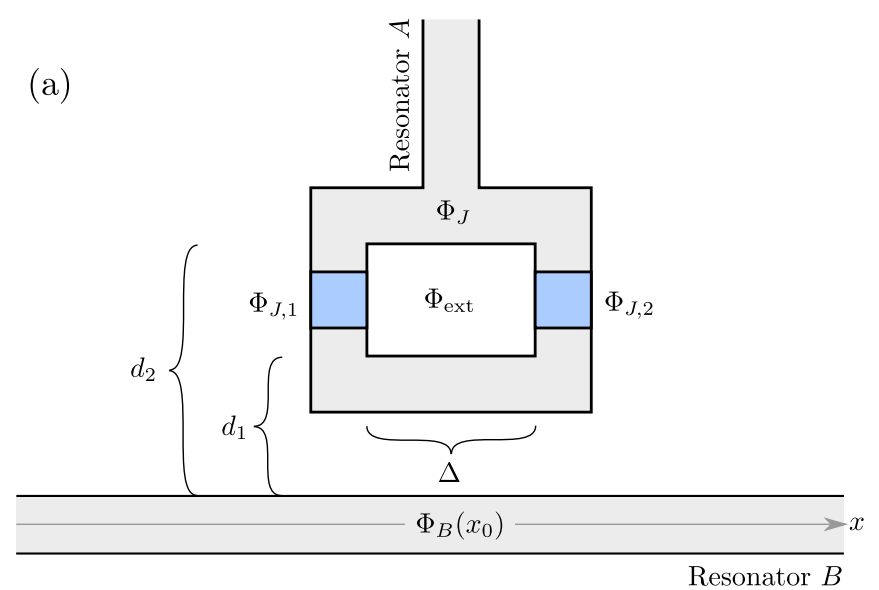

(b)

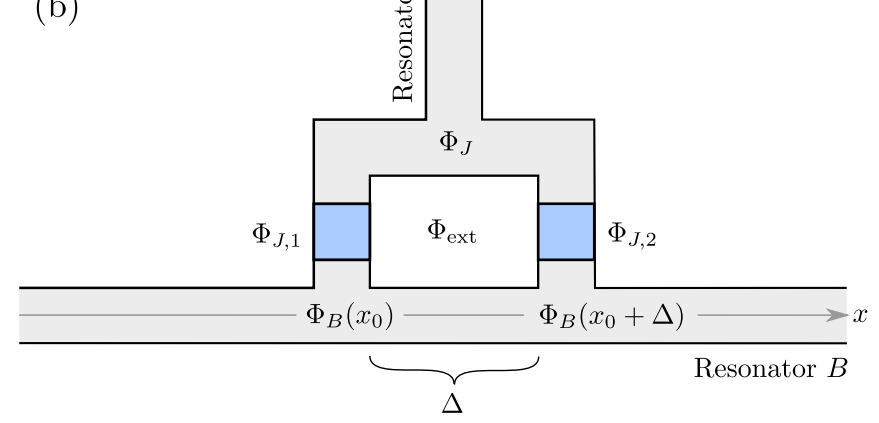

FIG. 4. (Color online) Two possible coupling geometries. (a) An inductive coupling design, where the magnetic field from resonator $B$ couples inductively to the SQUID loop. (b) An alternative coupling design, with potentially larger coupling strength, where the SQUID loop is galvanically connected to resonator $B$.

microstrips that define the microwave resonators. However, an estimate of the coupling strength can be obtained by assuming that the magnetic field $B(x, r)$ takes the form of that surrounding a perfect line conductor. In this case

$$
B(x, r)=\frac{\mu_{0} I_{B}(x)}{2 \pi r},
$$

where $r$ is the radial distance from the conductor, $I_{B}(x)$ is the current at position $x$, and $\mu_{0}$ is the permeability of free space. The current $I_{B}(x)$ can be evaluated using the expression for the field in terms of the mode functions, Eq. (24),

$$
\begin{aligned}
I_{B}\left(x_{0}\right)= & -L_{0}^{-1} \partial_{x} \Phi_{B}\left(x_{0}\right)=L_{0}^{-1} \sqrt{\frac{Z_{0} \hbar}{2 \pi}} \sum_{n} \sqrt{\frac{\omega_{d_{B}}}{\omega_{n}^{B}}} \frac{\pi n}{d_{B}} \\
& \times \sin \left(\frac{\pi n x_{0}}{d_{B}}\right)\left(b_{n} e^{-i \omega_{n}^{B} t}+\text { H.c. }\right) .
\end{aligned}
$$

The strongest coupling strength (for the fundamental mode $n=1$, as well as other odd- $n$ modes) is obtained by placing resonator $A$ so that it couples to resonator $B$ at the midpoint $x_{0}=d_{B} / 2$, in which case we have

$$
\begin{aligned}
I_{B}\left(x_{0}\right)= & L_{0}^{-1} \sqrt{\frac{Z_{0} \hbar}{2 \pi}} \frac{1}{d_{B}} \sum_{n} \sqrt{\frac{\omega_{d_{B}}}{\omega_{n}^{B}}} \pi n \\
& \times \sin \left(\frac{\pi n}{2}\right)\left(b_{n} e^{-i \omega_{n}^{B} t}+\text { H.c. }\right) .
\end{aligned}
$$

The magnetic flux through the SQUID due to the field from the resonator $B$ can be written

$$
\Delta \Phi_{\mathrm{ext}}=\int_{S} B d S=\int_{d_{1}}^{d_{2}} d r \int_{x_{0}}^{x_{0}+\Delta} d x B(x, r),
$$

and assuming that $I_{B}(x)$ is constant over $\left[x_{0}, x_{0}+\Delta\right]$, we obtain

$$
\Delta \Phi_{\mathrm{ext}}=\Delta \frac{\mu_{0} I_{B}\left(x_{0}\right)}{2 \pi} \int_{d_{1}}^{d_{2}} \frac{d r}{r}=\Delta \frac{\mu_{0} I_{B}\left(x_{0}\right)}{2 \pi} \ln \left(d_{2} / d_{1}\right) .
$$

Using the expression for the current given in Eq. (39), we have

$$
\begin{aligned}
\Delta \Phi_{\mathrm{ext}}= & \frac{\mu_{0} \Delta \ln \left(d_{2} / d_{1}\right)}{2 L_{0} d_{B}} \sqrt{\frac{Z_{0} \hbar}{2 \pi}} \sum_{n} \sqrt{\frac{\omega_{d_{B}}}{\omega_{n}^{B}}} n \\
& \times \sin \left(\frac{\pi n}{2}\right)\left(b_{n} e^{-i \omega_{n}^{B} t}+\text { H.c. }\right) .
\end{aligned}
$$

This can be written of the form of Eq. (28) with

$$
G_{n}^{\text {ind }}=\frac{\mu_{0} \Delta n \ln \left(d_{2} / d_{1}\right)}{2 L_{0} \Phi_{0} d_{B}} \sqrt{\frac{Z_{0} \hbar}{2 \pi} \frac{\omega_{d_{B}}}{\omega_{n}^{B}}} \sin \left(\frac{\pi n}{2}\right),
$$

and, in particular, for the fundamental mode $(n=1)$, in which we are most interested, we have

$$
G_{1}^{\text {ind }}=\mu_{0} L_{0}^{-1} \ln \left(d_{2} / d_{1}\right) \frac{\Delta}{2 d_{B}} \frac{1}{\Phi_{0}} \sqrt{\frac{Z_{0} \hbar}{2 \pi}} \sqrt{\frac{\omega_{d_{B}}}{\omega_{1}^{B}}} .
$$

\section{B. Galvanic coupling}

An alternative coupling design, which could produce stronger coupling, is shown in Fig. 4(b). In this case, part of the SQUID loop is galvanically connected to resonator $B$, and the fluxoid quantization condition for the SQUID loop is

$$
\Phi_{\mathrm{ext}}=\Phi_{J, 1}-\Phi_{J, 2}+\Phi_{B}\left(x_{0}+\Delta\right)-\Phi_{B}\left(x_{0}\right) .
$$

Assuming that the field in the resonator $B$ varies only slightly between $x_{0}$ and $x_{0}+\Delta$, we can linearize and write the difference $\Phi_{B}\left(x_{0}+\Delta\right)-\Phi_{B}\left(x_{0}\right)$ in Eq. (46) as a differential,

$$
\Phi_{\mathrm{ext}}=\Phi_{J, 1}-\Phi_{J, 2}+\Delta \partial_{x} \Phi_{B}\left(x_{0}\right) .
$$

We can now use this constraint to proceed as usual and eliminate one phase variable, and introduce the new variable $\Phi_{J}$ for the remaining SQUID degree of freedom

$$
\begin{aligned}
& \Phi_{J, 1}=\Phi_{J}+\frac{1}{2}\left[\Phi_{\mathrm{ext}}-\Delta \partial_{x} \Phi_{B}\left(x_{0}\right)\right], \\
& \Phi_{J, 2}=\Phi_{J}-\frac{1}{2}\left[\Phi_{\mathrm{ext}}-\Delta \partial_{x} \Phi_{B}\left(x_{0}\right)\right] .
\end{aligned}
$$

Here we identify $\Delta \Phi_{\text {ext }}=-\Delta \partial_{x} \Phi_{B}\left(x_{0}\right)$, and using the expression for the field of resonator $B$, Eq. (24), we obtain

$$
\begin{aligned}
\Delta \Phi_{\mathrm{ext}}= & \frac{\Delta}{d_{B}} \sqrt{\frac{Z_{0} \hbar}{2 \pi}} \sum_{n} \sqrt{\frac{\omega_{d_{B}}}{\omega_{n}^{B}}} \pi n \\
& \times \sin \left(\frac{\pi n x_{0}}{d_{B}}\right)\left(b_{n} e^{-i \omega_{n}^{B} t}+\text { H.c. }\right),
\end{aligned}
$$




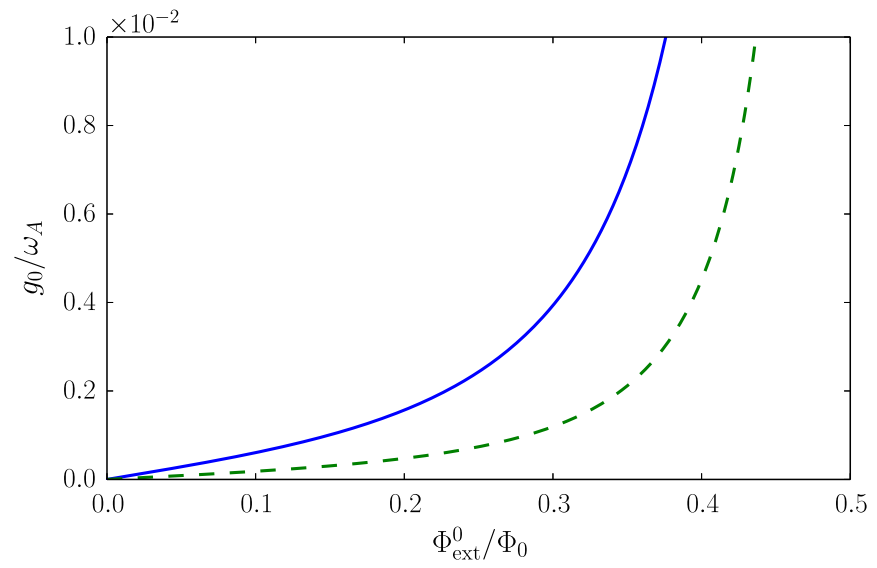

FIG. 5. (Color online) Normalized coupling strength $g_{0} / \omega_{A}$ as a function of the external flux bias $\Phi_{\text {ext }}$, for the galvanic (blue solid) and inductive (green dashed) coupling designs. The parameters used to evaluate Eq. (54) were $Z_{0} \approx 50 \Omega, \omega_{A}=10 \mathrm{GHz}, \omega_{B}=1 \mathrm{GHz}, d_{A}=$ $d_{B} / 20=3 \mathrm{~mm}, L_{0}=4.57 \times 10^{-7} \mathrm{H} / \mathrm{m}, C_{0}=1.46 \times 10^{-10} \mathrm{H} / \mathrm{m}$, $\Delta / d_{B}=10 \%$, and $E_{J}=4.11 \times 10^{-22} \mathrm{~J}$.

which can be written of the form of Eq. (28) with

$$
G_{n}^{\text {galv }}=\pi n \frac{\Delta}{d_{B}} \frac{1}{\Phi_{0}} \sqrt{\frac{Z_{0} \hbar}{2 \pi}} \sqrt{\frac{\omega_{d_{B}}}{\omega_{n}^{B}}} \sin \left(\frac{\pi n x_{0}}{d_{B}}\right) .
$$

Again, we are most interested in the coupling strength for the fundamental mode $(n=1)$, and for $x_{0}=d_{B} / 2$, we have

$$
G_{1}^{\mathrm{galv}}=\pi \frac{\Delta}{d_{B}} \frac{1}{\Phi_{0}} \sqrt{\frac{Z_{0} \hbar}{2 \pi}} \sqrt{\frac{\omega_{d_{B}}}{\omega_{1}^{B}}} .
$$

\section{Total coupling strength}

The ratio of the coupling strengths obtained for the inductive and galvanic coupling designs, assuming equal $\Delta$, is

$$
\frac{G_{1}^{\text {galv }}}{G_{1}^{\text {ind }}}=\frac{2 \pi}{\mu_{0} L_{0}^{-1} \ln \left(d_{2} / d_{1}\right)} .
$$

With $d_{2}=2 d_{1}, \mu_{0} \approx 1.26 \times 10^{-6} \mathrm{H} / \mathrm{m}$, and $L_{0}=4.57 \times$ $10^{-7} \mathrm{H} / \mathrm{m}$, this ratio is approximately 3.3 , demonstrating that galvanic coupling design is slightly more efficient.

The explicit form of the total coupling strength $g_{0}$, using the more favorable galvanic coupling design, is

$$
g_{0}=\omega_{1}^{A} \frac{\Delta}{d_{B}} \frac{\Delta d_{0}\left(\Phi_{\mathrm{ext}}^{0}\right)}{d_{A}+\Delta d_{0}\left(\Phi_{\mathrm{ext}}^{0}\right)} \frac{\pi^{2}}{\Phi_{0}} \sqrt{\frac{Z_{0} \hbar}{2 \pi} \frac{\omega_{d_{B}}}{\omega_{1}^{B}}} \tan \left(\pi \frac{\Phi_{\mathrm{ext}}^{0}}{\Phi_{0}}\right) .
$$

This expression is shown graphically in Figs. 5 and 6, for the specific parameters given in the captions. It is clear that $g_{0} \ll \omega_{A}$, as expected and required, but it is not necessary that $g_{0} \ll \omega_{B}$, since $\omega_{B}$ should be at least an order of magnitude smaller than $\omega_{A}$. Also, with resonators with sufficiently large $Q$ factors $\left(\sim 10^{3}\right)$, it should be possible to enter the strongcoupling regimes, where the frequency shift of resonator $A$ due to the presence of a single photon in resonator $B$ exceeds the linewidth of resonator $A$, i.e., $g_{0}>\kappa_{A}$, or when a single photon in resonator $A$ displaces resonator $B$ an amount that

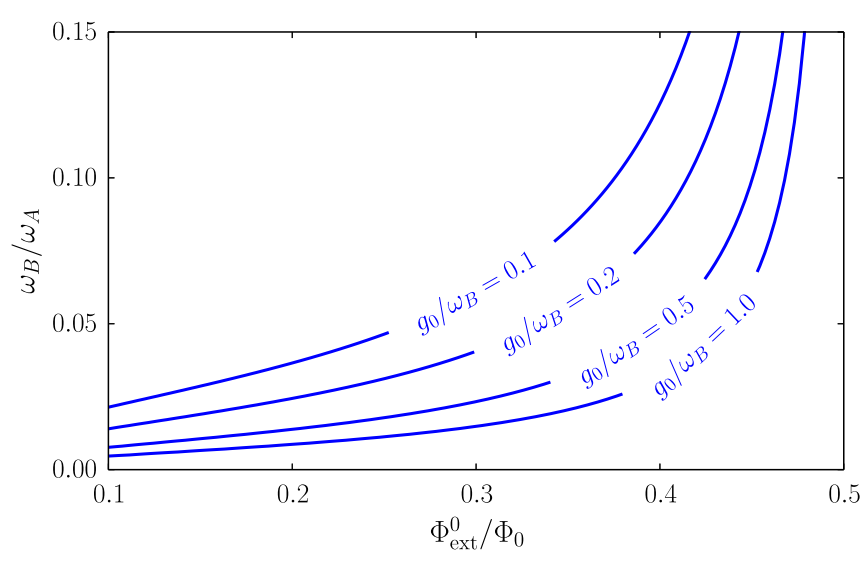

FIG. 6. (Color online) Contours of $g_{0} / \omega_{B}$, the ratio of the fundamental coupling strength to the frequency of resonator $B$, as a function of the ratio between the frequencies of resonator $B$ and $A$, $\omega_{B} / \omega_{A}$, and the external flux bias $\Phi_{\mathrm{ext}}^{0} / \Phi_{0}$, for the galvanic coupling design. Here we used the same parameters as in Fig. 5, except that here $\omega_{A}$ is varied.

exceeds its zero-point fluctuations, i.e., $g_{0}>\kappa_{B}$ and $g_{0} \sim \omega_{B}$. Here $\kappa_{A}$ and $\kappa_{B}$ denotes the relaxation rates of resonator $A$ and $B$, respectively.

In particular, the single-photon strong-coupling regime [7], where $g_{0} \sim \omega_{B}$, should be realizable in the circuit considered here. Figure 6 shows the ratio $g_{0} / \omega_{B}$ as a function of the resonator frequencies and the externally applied flux bias. When the frequency ratio $\omega_{B} / \omega_{A}$ is sufficiently small, it should be possible to reach $g_{0} \sim \omega_{B}$ for reasonable values of $\Phi_{\text {ext }}^{0} / \Phi_{0}$ (i.e., not too close to 0.5 ). The device we consider here is therefore a possible candidate for realizing an analog of an optomechanical system in this strong-coupling regime.

\section{DISSIPATION}

In the previous two sections we considered the effective Hamiltonian and estimated the achievable coupling strength between the two resonators. We noted that the single-photon coupling strength $g_{0}$ can be both tunable and strong, in the sense that it can be made comparable to the frequency of the resonator that plays the role of the mechanical resonator, $g_{0} \sim \omega_{B}$. However, the behavior of optomechanical systems, and their possible applications, is also strongly influenced by dissipation. In particular, to reach the resolved-sideband regime, the dissipation rates $\kappa_{A}$ and $\kappa_{B}$, of resonator $A$ and $B$, must not exceed the frequency of the "mechanical mode," i.e., $\kappa_{A}, \kappa_{B}<\omega_{B} / 2 \pi$. In most conventional optomechanical systems, the decay rate of the mechanical system is much smaller than the decay of the optical system, i.e., $\kappa_{B} \ll \kappa_{A}$. The relevant condition for the resolved-sideband regime is therefore $\kappa_{A}<\omega_{B} / 2 \pi$, and part of the challenge of reaching this regime is due to the fact that the optical and mechanical frequencies can be separated by many orders of magnitude. We note that in the circuit-QED realization considered here, the ratio $\omega_{A} / \omega_{B}$ is about 10 to 100 , which is much smaller than in a typical optomechanical system. It is therefore less challenging to reach the resolved sideband regime in the circuit-QED system introduced here. 
Thus we can choose the quality factors of superconducting transmission-line resonators, which are mainly determined by the capacitive coupling between the resonator and the external transmission line. These capacitors, denoted by $C_{A}$ and $C_{B}$ in Fig. 1, give rise to an external dissipation rate $\kappa_{\text {ext }}$. This is, of course, also the natural port for driving and reading out the state of the resonators. The internal dissipation, for example due to resistive and dielectric losses in the resonator, can be made small and the internal dissipation rate is thus usually negligible compared to the external dissipation rate, $\kappa_{\text {int }} \ll \kappa_{\text {ext }}$. The total dissipation rate of a resonator is therefore $\kappa=\kappa_{\mathrm{int}}+$ $\kappa_{\mathrm{ext}} \approx \kappa_{\mathrm{ext}}$. Experiments have demonstrated that quality factors exceeding 10000 are readily achievable [61]. Resonators that are terminated by a SQUID device, as is the case with resonator $A$, can in principle suffer from additional energy loss through the SQUID, corresponding to an increase of the internal decay rate. However, experiments have demonstrated that also this kind of device can be readily manufactured with quality factors between 5000 and 10000 [47], and that the quality factors of such resonators are not significantly decreased when the bias point is rapidly changed. This indicates that decay rates for the resonators considered here can be made much smaller than the frequencies of both resonators, $\kappa_{A}, \kappa_{B} \ll \omega_{A}, \omega_{B}$. However, the external decay rate can be designed in the fabrication process, and it is also possible to selectively make $\kappa_{A}$ or $\kappa_{B}$ larger or smaller than the relevant resonator frequencies, $\omega_{A}$ and $\omega_{B}$. It should therefore also be possible to realize, for example, the "reversed dissipation regime" (where $\kappa_{B} \gg \kappa_{A}$ ) considered in Ref. [62].

\section{ARRAYS OF COUPLED RESONATORS}

Using the nonlinear coupling mechanism for microwave resonators that we have investigated here, it is straightforward to imagine all-electrical networks, or arrays, of analog optomechanical resonators. Superstructures of optomechanical systems, for example optomechanical crystal arrays [63], have recently received considerable attention [64] for their potential applications in quantum information processing [65] and quantum simulation [66,67]. Implementing such systems with the all-electrical SQUID-coupled resonators considered here could have advantages in terms of designability, coupling strengths, and in situ controllability. Also, since all resonators in this architecture are electrical, they could all be probed and driven using the same microwave technologies. It is also relatively easy to construct various topologies among the coupled resonators, as we show below.

In a network of linearly coupled optomechanical systems, we can write the Hamiltonian for a single unit consisting of two nonlinearly coupled resonators as

$$
\begin{aligned}
H_{i} / \hbar= & \omega_{A}^{(i)} a_{i}^{\dagger} a_{i}+\omega_{B}^{(i)} b_{i}^{\dagger} b_{i}-g_{i} a_{i}^{\dagger} a_{i}\left(b_{i}+b_{i}^{\dagger}\right) \\
& +\epsilon_{A}^{(i)}\left(a_{i}+a_{i}^{\dagger}\right)+\epsilon_{B}^{(i)}\left(b_{i}+b_{i}^{\dagger}\right),
\end{aligned}
$$

where we have included driving fields applied to the two resonators, with amplitudes $\epsilon_{A}^{(i)}$ and $\epsilon_{B}^{(i)}$, for resonator $A$ and $B$, respectively. Apart from the additional driving fields, this Hamiltonian has the form of the effective Hamiltonian Eq. (30). The driving fields can be easily applied via the capacitive coupling to the external transmission lines.

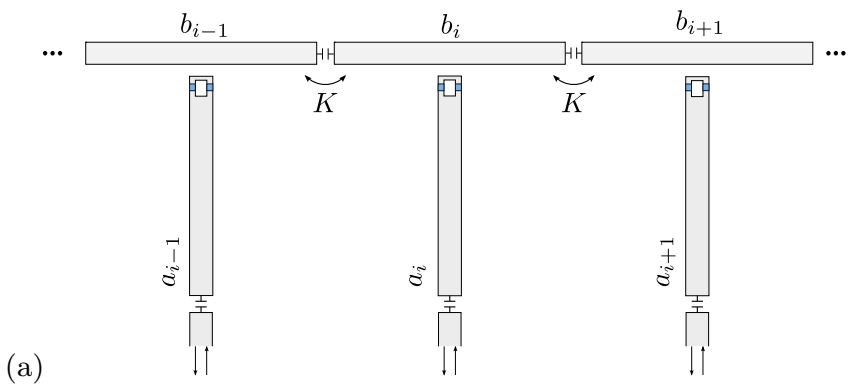

(b)
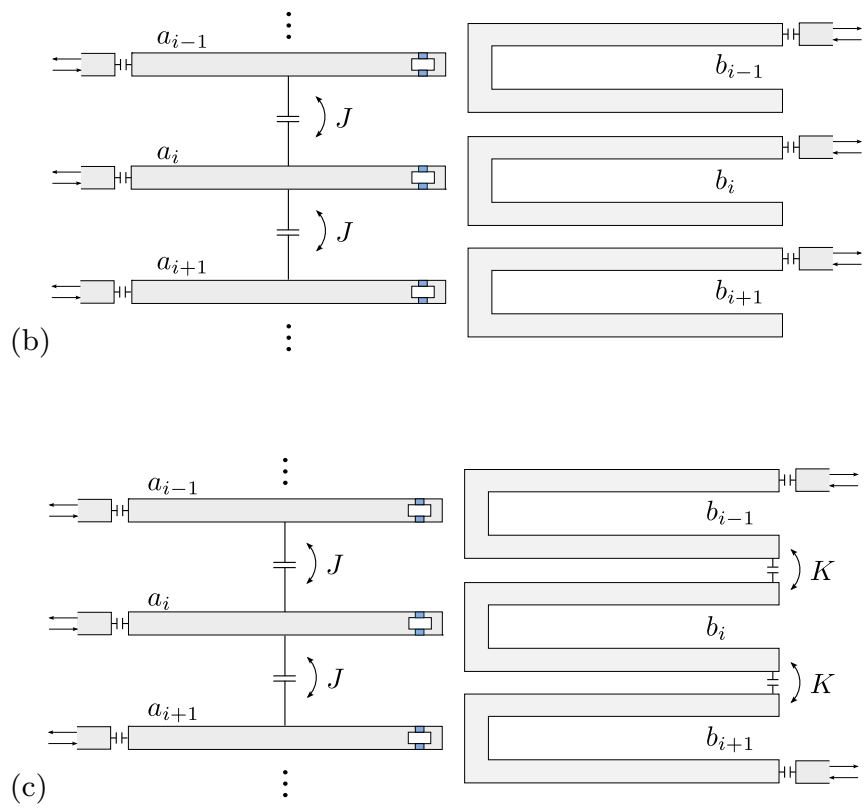

FIG. 7. (Color online) Three possible networks of nonlinearly coupled microwave resonators. These circuits represent all-electrical networks of resonators that are analogous to arrays of optomechanical systems. The electrical implementation here replaces the mechanical resonator with an electrical resonator, while keeping the nonlinear interaction. With different layouts it is possible to create networks where the "mechanical" (a), "optical" (b), or both (c) systems are coupled.

The Hamiltonian of a general linearly coupled nearestneighbor array of these unit systems can then be written of the form

$$
\begin{aligned}
H= & \sum_{i} H_{i}+J \sum_{\langle i, j\rangle}\left(a_{i}^{\dagger} a_{j}+a_{i} a_{j}^{\dagger}\right) \\
& +K \sum_{\langle i, j\rangle}\left(b_{i}^{\dagger} b_{j}+b_{i} b_{j}^{\dagger}\right) .
\end{aligned}
$$

Here $K$ and $J$ are the strengths of the linear coupling between resonators in different unit cells. In the case of electrical resonators, this type of coupling is realized using capacitive coupling between the resonators, and the strength of the coupling can be controlled in the design of the corresponding capacitances. Whether either or both of $K$ and $J$ are nonzero in a particular implementation depends on the layout. In Fig. 7, three possible layouts are shown schematically. The layout in Fig. 7(a) is a realization of a system described by the 
Hamiltonian Eq. (56) with $J=0$ and $K>0$ (i.e., coupled "mechanical" systems), Fig. 7(b) is a realization of a system with $K=0$ and $J>0$ (i.e., coupled "optical" systems), and Fig. 7(c) is a realization of a system where both $J>0$ and $K>0$ (i.e., both the mechanical and the optical systems are coupled).

\section{CONCLUSIONS}

We have introduced and analyzed a nonlinear coupling mechanism for superconducting microwave resonators. With the proposed coupling scheme, it is possible to realize analogs of optomechanical systems in an all-electrical circuit. The optomechanical-like interaction can be made both strong and tunable through an externally applied flux bias. This all-electrical realization of optomechanical-like systems could therefore be used to explore the optomechanical model in new interesting regimes. We have also discussed potential applications of this circuit realization of the optomechanical model as an alternative way of implementing arrays of "optomechanical" systems, which can be used, for example, in quantum simulator applications. We believe that the introduced nonlinear coupling provides new opportunities for implementing analogs of quantum systems in superconducting circuits.

\section{ACKNOWLEDGMENTS}

We wish to thank X.-Y. Lü, H. Jing, J. Bylander, and E.-J. Kim for helpful discussions. This work was partly supported by the RIKEN iTHES Project, MURI Center for Dynamic Magneto-Optics, JSPS-RFBR No. 12-02-92100, and a Grantin-Aid for Scientific Research (S). G.J. acknowledges funding from the Swedish Research Council and from the EU through the ERC and the projects PROMISCE and SCALEQIT.
[1] J. Q. You and F. Nori, Phys. Today 58, 42 (2005).

[2] J. Clarke and F. K. Wilhelm, Nature (London) 453, 1031 (2008).

[3] J. Q. You and F. Nori, Nature (London) 474, 589 (2011).

[4] M. H. Devoret and R. J. Schoelkopf, Science 339, 1169 (2013).

[5] M. Poot and H. S. van der Zant, Phys. Rep. 511, 273 (2012).

[6] P. Meystre, Ann. Phys. (Leipzig) 525, 215 (2013).

[7] M. Aspelmeyer, T. J. Kippenberg, and F. Marquardt, arXiv:1303.0733.

[8] J. D. Teufel, T. Donner, D. Li, J. W. Harlow, M. S. Allman, K. Cicak, A. J. Sirois, J. D. Whittaker, K. W. Lehnert, and R. W. Simmonds, Nature (London) 475, 359 (2011).

[9] J. Chan, T. P. M. Alegre, A. H. Safavi-Naeini, J. T. Hill, A. Krause, S. Groblacher, M. Aspelmeyer, and O. Painter, Nature (London) 478, 89 (2011).

[10] S. Groblacher, K. Hammerer, M. R. Vanner, and M. Aspelmeyer, Nature (London) 460, 724 (2009).

[11] J. D. Teufel, D. Li, M. S. Allman, K. Cicak, A. J. Sirois, J. D. Whittaker, and R. W. Simmonds, Nature (London) 471, 204 (2011).

[12] E. Verhagen, S. Deleglise, S. Weis, A. Schliesser, and T. J. Kippenberg, Nature (London) 482, 63 (2012).

[13] D. W. C. Brooks, T. Botter, S. Schreppler, T. P. Purdy, N. Brahms, and D. M. Stamper-Kurn, Nature (London) 488, 476 (2012).

[14] A. H. Safavi-Naeini, S. Groblacher, J. T. Hill, J. Chan, M. Aspelmeyer, and O. Painter, Nature (London) 500, 185 (2013).

[15] C. A. Regal, J. D. Teufel, and K. W. Lehnert, Nat. Phys. 4, 555 (2008).

[16] J. Teufel, T. Donner, M. Castellanos-Beltran, J. Harlow, and K. Lehnert, Nat. Nanotechnol. 4, 820 (2009).

[17] J. B. Hertzberg, T. Rocheleau, T. Ndukum, M. Savva, A. A. Clerk, and K. C. Schwab, Nat. Phys. 6, 213 (2009).

[18] T. A. Palomaki, J. W. Harlow, J. D. Teufel, R. W. Simmonds, and K. W. Lehnert, Nature (London) 495, 210 (2013).

[19] T. A. Palomaki, J. D. Teufel, R. W. Simmonds, and K. W. Lehnert, Science 342, 710 (2013).

[20] J. Q. You and F. Nori, Phys. Rev. B 68, 064509 (2003).

[21] A. Blais, R.-S. Huang, A. Wallraff, S. M. Girvin, and R. J. Schoelkopf, Phys. Rev. A 69, 062320 (2004).
[22] R. J. Schoelkopf and S. M. Girvin, Nature (London) 451, 664 (2008).

[23] T. Duty, G. Johansson, K. Bladh, D. Gunnarsson, C. Wilson, and P. Delsing, Phys. Rev. Lett. 95, 206807 (2005).

[24] A. Wallraff, D. I. Schuster, A. Blais, L. Frunzio, J. Majer, M. H. Devoret, S. M. Girvin, and R. J. Schoelkopf, Phys. Rev. Lett. 95, 060501 (2005).

[25] I. Siddiqi, R. Vijay, M. Metcalfe, E. Boaknin, L. Frunzio, R. J. Schoelkopf, and M. H. Devoret, Phys. Rev. B 73, 054510 (2006).

[26] A. Lupaşcu, E. F. C. Driessen, L. Roschier, C. J. P. M. Harmans, and J. E. Mooij, Phys. Rev. Lett. 96, 127003 (2006).

[27] M. R. Delbecq, V. Schmitt, F. D. Parmentier, N. Roch, J. J. Viennot, G. Fève, B. Huard, C. Mora, A. Cottet, and T. Kontos, Phys. Rev. Lett. 107, 256804 (2011).

[28] S. J. Chorley, J. Wabnig, Z. V. Penfold-Fitch, K. D. Petersson, J. Frake, C. G. Smith, and M. R. Buitelaar, Phys. Rev. Lett. 108, 036802 (2012).

[29] T. Frey, P. J. Leek, M. Beck, A. Blais, T. Ihn, K. Ensslin, and A. Wallraff, Phys. Rev. Lett. 108, 046807 (2012).

[30] J. I. Colless, A. C. Mahoney, J. M. Hornibrook, A. C. Doherty, H. Lu, A. C. Gossard, and D. J. Reilly, Phys. Rev. Lett. 110, 046805 (2013).

[31] Z.-L. Xiang, S. Ashhab, J. Q. You, and F. Nori, Rev. Mod. Phys. 85, 623 (2013).

[32] L. Zhou, Z. R. Gong, Y.-X. Liu, C. P. Sun, and F. Nori, Phys. Rev. Lett. 101, 100501 (2008).

[33] F. Helmer, M. Mariantoni, A. G. Fowler, J. von Delft, E. Solano, and F. Marquardt, Europhys. Lett. 85, 50007 (2009).

[34] S. Schmidt, D. Gerace, A. A. Houck, G. Blatter, and H. E. Türeci, Phys. Rev. B 82, 100507 (2010).

[35] D. L. Underwood, W. E. Shanks, J. Koch, and A. A. Houck, Phys. Rev. A 86, 023837 (2012).

[36] B. Peropadre, D. Zueco, F. Wulschner, F. Deppe, A. Marx, R. Gross, and J. J. Garcia-Ripoll, Phys. Rev. B 87, 134504 (2013).

[37] P. Rabl, Phys. Rev. Lett. 107, 063601 (2011).

[38] A. Nunnenkamp, K. Børkje, and S. M. Girvin, Phys. Rev. Lett. 107, 063602 (2011). 
[39] A. Nunnenkamp, K. Børkje, and S. M. Girvin, Phys. Rev. A 85, 051803 (2012).

[40] S. Bose, K. Jacobs, and P. L. Knight, Phys. Rev. A 56, 4175 (1997).

[41] J. Qian, A. A. Clerk, K. Hammerer, and F. Marquardt, Phys. Rev. Lett. 109, 253601 (2012).

[42] A. Kronwald, M. Ludwig, and F. Marquardt, Phys. Rev. A 87, 013847 (2013).

[43] P. D. Nation, Phys. Rev. A 88, 053828 (2013).

[44] M. Wallquist, V. S. Shumeiko, and G. Wendin, Phys. Rev. B 74, 224506 (2006).

[45] L. Chirolli, G. Burkard, S. Kumar, and D. P. DiVincenzo, Phys Rev. Lett. 104, 230502 (2010).

[46] A. Palacios-Laloy, F. Nguyen, F. Mallet, P. Bertet, D. Vion, and D. Esteve, J. Low Temp. Phys. 151, 1034 (2008).

[47] M. Sandberg, C. M. Wilson, F. Persson, T. Bauch, G. Johansson, V. Shumeiko, T. Duty, and P. Delsing, Appl. Phys. Lett. 92, 203501 (2008).

[48] T. Yamamoto, K. Inomata, M. Watanabe, K. Matsuba, T. Miyazaki, W. D. Oliver, Y. Nakamura, and J. S. Tsai, Appl. Phys. Lett. 93, 042510 (2008).

[49] M. A. Castellanos-Beltran, K. D. Irwin, G. C. Hilton, L. R. Vale, and K. W. Lehnert, Nat. Phys. 4, 929 (2008).

[50] C. M. Wilson, T. Duty, M. Sandberg, F. Persson, V. Shumeiko, and P. Delsing, Phys. Rev. Lett. 105, 233907 (2010).

[51] J. R. Johansson, G. Johansson, C. M. Wilson, and F. Nori, Phys. Rev. Lett. 103, 147003 (2009).

[52] J. R. Johansson, G. Johansson, C. M. Wilson, and F. Nori, Phys. Rev. A 82, 052509 (2010).
[53] J. R. Johansson, G. Johansson, C. M. Wilson, P. Delsing, and F. Nori, Phys. Rev. A 87, 043804 (2013).

[54] C. M. Wilson, G. Johansson, A. Pourkabirian, M. Simonen, J. R. Johansson, T. Duty, F. Nori, and P. Delsing, Nature (London) 479, 376 (2011).

[55] P. Lähteenmäki, G. S. Paraoanu, J. Hassel, and P. J. Hakonen, Proc. Natl. Acad. Sci. USA 110, 4234 (2013).

[56] P. D. Nation, J. R. Johansson, M. P. Blencowe, and F. Nori, Rev. Mod. Phys. 84, 1 (2012).

[57] B. Yurke and J. S. Denker, Phys. Rev. A 29, 1419 (1984).

[58] M. Devoret, Quantum Fluctuations in Electrical Circuits, Les Houches LXIII (Elsevier, Amsterdam, 1995).

[59] S. Mancini and P. Tombesi, Phys. Rev. A 49, 4055 (1994).

[60] X.-Y. Lü, W.-M. Zhang, S. Ashhab, Y. Wu, and F. Nori, Sci. Rep. 3, 2943 (2013).

[61] M. Göppl, A. Fragner, M. Baur, R. Bianchetti, S. Filipp, J. M. Fink, P. J. Leek, G. Puebla, L. Steffen, and A. Wallraff, J. Appl. Phys. 104, 113904 (2008).

[62] A. Nunnenkamp, V. Sudhir, A. K. Feofanov, A. Roulet, and T. J. Kippenberg, Phys. Rev. Lett. 113, 023604 (2014).

[63] D. E. Chang, A. H. Safavi-Naeini, M. Hafezi, and O. Painter, New J. Phys. 13, 023003 (2011).

[64] A. Xuereb, C. Genes, and A. Dantan, Phys. Rev. Lett. 109, 223601 (2012).

[65] M. Schmidt, M. Ludwig, and F. Marquardt, New J. Phys. 14, 125005 (2012).

[66] A. Tomadin, S. Diehl, M. D. Lukin, P. Rabl, and P. Zoller, Phys. Rev. A 86, 033821 (2012).

[67] M. Ludwig and F. Marquardt, Phys. Rev. Lett. 111, 073603 (2013). 